\title{
Indicadores de síntomas de abstinencia en un grupo de fumadores mexicanos
}

\author{
Gabriela Lara-Rivas, Lic en Psic, ${ }^{(1)}$ Alejandra Ramírez-Venegas, M en C, ${ }^{(1)}$ Raúl H Sansores-Martínez, M en C, (2) \\ Ana Marlene Espinosa, Lic Psic, ${ }^{(1)}$ Justino Regalado-Pineda, M en C. ${ }^{(1)}$
}

Lara-Rivas G, Ramírez-Venegas A, Sansores-Marínez RH,
Espinosa AM, Regalado-Pineda J.
Indicadores de síntomas de abstinencia
en un grupo de fumadores mexicanos.
Salud Publica Mex 2007;49 supl 2:S257-S262.

\section{Resumen}

Objetivo. Identificar indicadores de abstinencia en fumadores de la Clínica Anti-Tabaco en un hospital de referencia. Evaluar los síntomas según el nivel de adicción a la nicotina mediante la escala de Fagerström (EDNF). Material y métodos. Se incluyeron fumadores de la Clínica Anti-Tabaco y no-fumadores visitantes. Fumadores y no-fumadores completaron una entrevista estandarizada y diario de síntomas. Resultados. Se incluyeron I 85 fumadores (edad 40.93 años, DE, I0.22, 93 hombres/92 mujeres) y 102 no-fumadores (edad 38.22 años, DE 9.40,4I hombres/6I mujeres). La mediana del índice paquetes-año fue de I3 (rango I-72), el promedio de la EDNF fue de 6 (DE, 2.35). Los síntomas asociados con la dependencia fueron: ansiedad, RM I.2I (IC95\% I.03-I.43); depresión, RM 2.75 (IC95\%।.07-7.04), la depresión es más común entre las mujeres, RM 2.03 (IC95\% I.I0 - 3.75); deseos de fumar, RM I.53 (IC95\% I.19-I.97) y sudoración, RM 2.73 (IC95\% I.07-6.95).Conclusiones. Los indicadores de abstinencia de nicotina en un grupo de fumadores mexicanos fueron deseos de fumar, ansiedad, depresión y diaforesis; estos síntomas se correlacionan con el nivel de dependencia a la nicotina.

Palabras clave: Síndrome de Abstinencia; Dejar de Fumar; Fumadores mexicanos
Lara-Rivas G, Ramírez-Venegas A, Sansores-Marínez RH, Espinosa AM, Regalado-Pineda J. Withdrawal symptoms indicators in a group of mexican smokers. Salud Publica Mex 2007;49 suppl 2:S257-S262.

\section{Abstract}

Objective. To describe symptoms of AS in a group of Mexican smokers attending a third referral clinic, to evaluate symptoms according to degree of nicotine dependence using the Fagerström Test (FTND). Material and Methods. A self-reported standardized questionnaire was administered to smokers and non-smokers during three consecutive days. None of the smokers received treatment during this period. Results. A total of I 85 smokers and 102 non-smokers were included. No differences in age or gender were observed. Among smokers, median tobacco consumption was 13 pack-years (range I to 72) and the mean (SD) FTND was six (2.35). Symptoms related to nicotine withdrawal were anxiety (OR I.2I, 95\%IC I.03-I.43), depression (OR 2.75, 95\%IC I.07-7.04), which was more common in women (OR 2.03, 95\%IC I.I0-3.75), urge to smoke (OR I.53, 95\%IC I.I9-I.97), and sweating (OR 2.73, 95\%IC I.07-6.95). Conclusions. The most frequent symptoms related to nicotine abstinence in Mexican smokers were: anxiety, urge to smoke, depression and sweating. These symptoms correlate with the degree of addiction.

Key words:Withdrawal syndrome; smoking cessation; Mexican smokers

(I) Departamento de Investigación en Tabaquismo. Instituto Nacional de Enfermedades Respiratorias. México.

(2) Unidad de Investigación. Instituto Nacional de Enfermedades Respiratorias. México.

Fecha de recibido: 25 de abril de 2007 - Fecha de aceptado: 14 de mayo de 2007

Solicitud de sobretiros: Lic.A. Gabriela Lara Rivas. Departamento de Investigación en Tabaquismo. Instituto Nacional de Enfermedades Respiratorias, Secretaría de Salud. Calzada de Tlalpan 4502. 14080 México, DF, México.

Correo electrónico: agabilara@yahoo.com.mx 
E

tabaquismo constituye en la actualidad uno de los problemas de salud más importantes a nivel mundial; de acuerdo con los datos de la Organización Mundial de la Salud (OMS), la prevalencia del tabaquismo es de aproximadamente $30 \% .^{1}$ Solamente en México ocurren más de 60000 muertes al año entre personas fumadoras por enfermedades asociadas al tabaquismo. ${ }^{2}$

La Encuesta Nacional de Adicciones 2002 (ENA 2002) reporta que la prevalencia nacional de consumo de cigarrillos en la población urbana es de entre 12 y 65 años de edad, lo que representa casi 16 millones de individuos fumadores ( $26.4 \%$ de la población mexicana de 12 a 65 años). ${ }^{3}$ El tabaquismo se encuentra relacionado de manera causal con múltiples enfermedades, muchas de ellas discapacitantes y mortales, ${ }^{4}$ y se asocia con una notable disminución en la sobrevida y pérdida de años de vida saludable (en promedio 20 a 25 años). ${ }^{3}$ Se han identificado, hasta este momento, 26 enfermedades asociadas a esta adicción. 4,5

La nicotina constituye el componente bioactivo contenido en el humo del cigarrillo y resulta la sustancia responsable del desarrollo de adicción y dependencia, fenómeno observado tanto en humanos como en modelos animales. ${ }^{6-8}$ Las consecuencias por la interrupción abrupta de la administración de la nicotina se conocen desde hace tiempo; dejar de fumar repentinamente conlleva una serie de s ntomas cuyo conjunto conforma el s ndrome de abstinencia. ${ }^{9}$ Desde su descripción original a mediados de la década de 1940, se han llevado a cabo un número considerable de estudios internacionales para determinar los s ntomas de abstinencia asociados con dejar de fumar. ${ }^{10,11}$ La prevalencia, intensidad y tiempo de aparición de estos s ntomas var an de una persona a otra y son una de las razones principales por las que un fumador recae y vuelve a fumar. ${ }^{12}$

La Clasificación Internacional de las Enfermedades, décima edición (CIE-10), considera al s ndrome de abstinencia como una entidad aparte en relación con la suspensión en el consumo de sustancias. ${ }^{13}$ De acuerdo al Manual Estad stico y Diagnóstico de enfermedades siquiátricas (DSM- IV) $)^{8}$ el s ndrome de abstinencia incluye cuatro o más de los siguientes s ntomas: estados de ánimo disfórico o depresivo, insomnio, irritabilidad, frustración o ira, ansiedad, dificultad de concentración, inquietud o impaciencia, disminución de la frecuencia cardiaca, aumento del apetito o aumento del peso. Los s ntomas de abstinencia provocan un malestar cl nicamente significativo o deterioro laboral, social o de otras áreas importantes de la actividad del individuo. Estos s ntomas no se asocian de manera causal con otras condiciones médicas ni se explican por la presencia de otros trastornos mentales. ${ }^{8}$
No existe evidencia en la literatura nacional o internacional de estudios que describan las caracter sticas del s ndrome de abstinencia de la nicotina en población mexicana. El presente trabajo se realizó con el objetivo de describir las caracter sticas del s ndrome de abstinencia en un grupo de pacientes que acudieron para recibir tratamiento en la cl nica de ayuda para dejar de fumar del Instituto Nacional de Enfermedades Respiratorias Ismael Cos o Villegas, INER.

\section{Material y métodos}

Se seleccionaron pacientes consecutivos que acudieron a la cl nica para dejar de fumar del INER y que aceptaron participar en el estudio previa firma de carta de consentimiento informado. Para la selección de pacientes se emplearon los siguientes criterios de inclusión: a) fumadores mayores de 18 años de edad; b) consumo de al menos 10 cigarrillos diarios; c) hombres o mujeres; d) registrados en el programa para dejar de fumar del INER; e) sin haber recibido tratamiento farmacológico o psicológico para dejar de fumar en los tres meses previos; f) sin consumo de alcohol $u$ otras substancias ilegales como marihuana, coca na o hero na; g) sin enfermedades psiquiátricas diagnosticadas por médico de acuerdo al Manual de Diagnóstico Estad stico de Enfermedades Mentales, versión IV (DSM IV).

Con la finalidad de comparar la prevalencia de s ntomas potencialmente relacionados con s ndrome de abstinencia se seleccionaron visitantes no fumadores como grupo control, los cuales también firmaron una carta de consentimiento informado. Se invitó a participar en el estudio a personas que acud an como visitantes al INER, ya fuera acompañando a pacientes que acud an a la cl nica de ayuda para dejar de fumar solicitando información, o bien como visitantes de pacientes internados en el INER por cualquiera de las enfermedades que se atienden. Para la selección de los no fumadores se aplicaron los siguientes criterios de inclusión: a) sujetos mayores de 18 años de edad; b) ambos sexos; c) nunca fumadores o ex fumadores con más de 5 años de abstinencia continua confirmada mediante prueba de monóxido de carbono (CO) al final de la espiración; d) sin consumo de alcohol u otras substancias ilegales; y e) sin enfermedades psiquiátricas. No se realizó ningún tipo de pareamiento entre el grupo de fumadores y el grupo de no fumadores. Cada paciente fumador y su control completó una entrevista psiquiátrica estandarizada ${ }^{14}$ para descartar algún tipo de padecimiento psiquiátrico, as como el consumo de otras sustancias como alcohol, marihuana, coca na, etc.; además, completaron el Cuestionario de Fagerström, para conocer el grado de dependencia a la nicotina. ${ }^{14}$ 
Se registraron s ntomas asociados con el s ndrome de abstinencia, tomando en cuenta diferentes instrumentos, as como los elementos considerados para el diagnóstico de s ndrome de abstinencia en el DSM-IV. ${ }^{8}$ Mediante diario de autorreporte se registraron diferentes s ntomas durante los primeros tres $\mathrm{d}$ as a partir de haber dejado de fumar, en el caso de los pacientes, y a partir de ser incluidos en el estudio, en el caso de los no fumadores. Durante este periodo ninguno de los pacientes del grupo de fumadores recibió tratamiento médico o psicológico. El estudio fue evaluado y aprobado por el Comité de Ciencia y Bioética del INER.

En relación con el análisis estad stico, las variables con distribución normal se establecieron mediante prueba de $t$ de Student para diferencias de promedios entre los dos grupos. Las variables dicotómicas fueron analizadas mediante diferencias de proporciones y prueba de ji cuadrada o Prueba Exacta de Fisher, según el caso, entre fumadores y controles. Para determinar la asociación de las distintas variables incluidas en el diario de s ntomas con el hecho de ser fumador o control se realizó un análisis de regresión log stica para obtener la razón de probabilidad (RM) ajustando por edad, género y nivel de dependencia a la nicotina. Se emplearon programas del paquete estad stico Stata versión 8.0 .

\section{Resultados}

De enero a noviembre de 2003 se incluyeron 185 pacientes fumadores consecutivos que acudieron a la cl nica de ayuda para dejar de fumar del INER con la intención de recibir tratamiento y a 102 no fumadores que aceptaron participar en el estudio. Tanto los fumadores como los no fumadores completaron un diario de autorreporte de $\mathrm{s}$ ntomas durante los primeros tres $\mathrm{d}$ as posteriores al abandono del tabaco entre los fumadores y, en el caso de los segundos, al ser reclutados el estudio. La tasa de aceptación entre los fumadores se considera aceptable; ninguno de los fumadores registrados en el programa para dejar de fumar del INER se negó a completar el diario de s ntomas. Por su parte, los individuos del grupo de no fumadores presentaron una tasa de respuesta de 87 por ciento.

El diario de autorreporte conten a una lista con 18 s ntomas asociados al s ndrome de abstinencia: ansiedad, tensión, depresión, hipersomnia, insomnio, malestar gastrointestinal, urticaria, falta de concentración, irritabilidad, aumento del apetito, palpitaciones, cambios en la presión sangu nea, cefalea, sudoración, temblor, mareo, hormigueos, deseos de fumar. Los s ntomas fueron categorizados por los pacientes en leve, moderado o severo, según su intensidad.
En el cuadro I se presentan las caracter sticas generales de los fumadores y no fumadores. El promedio de edad de los fumadores fue de 40.93 años (Desviación Estándar (DE) 10.22); el promedio de edad de los controles fue de 38.22 años (DE 9.40); no se encontraron diferencias significativas en términos de edad. En el grupo de pacientes se incluyeron 93 hombres y 92 mujeres, y en el grupo de pacientes no fumadores se incluyeron 41 hombres y 61 mujeres. No se observaron diferencias de proporciones en relación con el género (ji cuadrada 2.68, p=0.102). Los fumadores presentaban una mediana del ndice de tabaquismo de 13 paquetesaño, rango de 1 a 72. En términos de dependencia a la nicotina, la mediana de la Escala de Dependencia a la Nicotina de Fagerström (EDNF) fue de 6 (rango 0-10).

Cuadro I

Características generales de los participantes. InStituto NaCional de ENFERMEdAdES Respiratorias. MÉXICO, 2003

\begin{tabular}{|c|c|c|}
\hline & $\begin{array}{c}\text { Fumadores } \\
n=185\end{array}$ & $\begin{array}{c}\text { No fumadores } \\
n=102\end{array}$ \\
\hline Edad, promedio (DE) & $40(0.75)$ & $38.21(0.93)^{*}$ \\
\hline Hombres/Mujeres & $93 / 92$ & $4 \mid / 6 I$ \\
\hline \multicolumn{3}{|l|}{ Paquetes-año, } \\
\hline mediana (rango) & $13(1-72)$ & $0(0-0)$ \\
\hline \multicolumn{3}{|l|}{ Prueba de dependencia } \\
\hline de Fagerström mediana (rango) & $6(0,10)$ & $0(0,0)$ \\
\hline Deseos de fumar, $n(\%)$ & 169 (9I.35) & $0(0)^{\ddagger}$ \\
\hline Ansiedad, n (\%) & $147(79.46)$ & $26(25.49)^{\S}$ \\
\hline Aumento del apetito, n (\%) & $119(64.32)$ & $0(0)^{\ddagger}$ \\
\hline Irritabilidad, n (\%) & $113(61.08)$ & $0(0)^{\ddagger}$ \\
\hline Tensión, n (\%) & $109(58.92)$ & $33(32.35)^{\S}$ \\
\hline Depresión, n (\%) & $91(41.19)$ & $29(28.43)^{\S}$ \\
\hline Insomnio, $\mathrm{n}(\%)$ & $85(45.95)$ & II $(10.78)^{\S}$ \\
\hline Malestar gastrointestinal, $\mathrm{n}(\%)$ & $80(43.24)$ & $22(21.57)^{\S}$ \\
\hline Mareo, n (\%) & $76(4 I .08)$ & $0(0)^{\ddagger}$ \\
\hline Cefalea, n (\%) & $72(38.92)$ & $0(0)^{\ddagger}$ \\
\hline Dificultad para concentrarse, $\mathrm{n}(\%)$ & $72(38.92)$ & $9(8.82)^{\S}$ \\
\hline Sudoración, n (\%) & $68(36.76)$ & $0(0)^{\ddagger}$ \\
\hline Palpitaciones, n (\%) & $59(31.89)$ & $0(0)^{\ddagger}$ \\
\hline Temblor, n (\%) & $56(30.27)$ & $0(0)^{\ddagger}$ \\
\hline Hormigueo, n (\%) & $49(26.49)$ & $0(0)^{\ddagger}$ \\
\hline Hipersomnia, n (\%) & $69(37.30)$ & $34(33.33)$ \\
\hline Urticaria, n (\%) & $19(10.27)$ & $8(7.84)$ \\
\hline \multicolumn{3}{|l|}{${ }^{*} p>0.05$ por Prueba de $t$ de Student } \\
\hline \multicolumn{3}{|l|}{$\neq p<0.05$ por Prueba Exacta de Fisher } \\
\hline$\S p<0.05$ por prueba de ji cuadrada & & \\
\hline
\end{tabular}

Los participantes fueron reclutados entre enero y noviembre de 2003 en la clínica para dejar de fumar del Instituto Nacional de Enfermedades Respiratorias (INER), México. 
En $25 \%$ de los pacientes fumadores se documentó una puntuación EDNF de 0 a 4 puntos y 25\% de los fumadores reportó una puntuación de 8 o más puntos en la EDNF. Se observó mayor frecuencia de reporte de s ntomas entre los sujetos del grupo de fumadores en comparación con los no fumadores para los siguientes s ntomas explorados: ansiedad, tensión, depresión, insomnio, malestar gastrointestinal y dificultad para concentrarse, $p<0.05$ por prueba de ji cuadrada. En el caso de los tems correspondientes a irritabilidad, aumento del apetito, palpitaciones, temblor, mareo, hormigueo, deseos de fumar, cefalea y sudoración, se observó que ninguno de los sujetos del grupo de no fumadores registró en el diario de autorreporte alguna de las variables consideradas, $p<0.05$ por prueba exacta de Fisher (cuadro I).

Con la finalidad de explorar la posible asociación entre los s ntomas que resultaron diferentes entre fumadores y no fumadores, se realizó un análisis de regresión log stica entre los sujetos del grupo de fumadores ajustando por edad, género y nivel de abstinencia a la nicotina como variable continua, y como variable de contraste comparando el nivel de adicción baja a moderada (EDNF 0 a 4) con un nivel de adicción intenso $(E D N F>6)$. En el cuadro II se muestra la RM y los intervalos de confianza al 95\% de los modelos obtenidos en el grupo de fumadores. La ansiedad se asocia significativamente con el nivel de adicción a la nicotina, RM 1.21 (IC95\% 1.03 - 1.43). La probabilidad de desarrollar depresión al dejar de fumar se asocia de manera independiente con el nivel de adicción, especialmente cuando la escala de Fagerström supera los 6 puntos, RM 2.75 (IC95\% 1.07 - 7.04). En el caso de s ntomas de depresión se observó que ésta es más común entre las mujeres, RM 2.03 (IC95\% 1.10 - 3.75). La probabilidad de desarrollar molestias o malestar gastrointestinal as como aumento del apetito se asocia con menor edad, es decir, la probabilidad de presentar malestar gastrointestinal o aumento del apetito al dejar de fumar se reduce al aumentar la edad, RM 0.96 (IC95\% 0.93 - 0.99) y RM 0.96 (IC95\% 0.93 - 0.99) respectivamente. La aparición de deseos de fumar se asocia positivamente con el nivel de adicción a la nicotina, RM 1.53 (IC95\% 1.19 - 1.97). Finalmente, la sudoración o diaforesis se asocia con la adicción a la nicotina, particularmente cuando la prueba de dependencia de Fagerström es mayor de 6 puntos, RM 2.73 (IC95\% 1.07 - 6.95). En la figura 1 se muestra la asociación entre la ansiedad, depresión, deseos de fumar y diaforesis con el nivel de dependencia a la nicotina. Algunas variables que en el análisis univariado hab an mostrado mayor prevalencia entre sujetos fumadores (como tensión, hipersomnia, insomnio, dificultad para

\section{Cuadro II}

Modelos de Regresión logística en el GRUPO de FUMADORES, AJUSTANDO POR EDAD, GÉNERO Y NIVEL DE DEPENDENCIA A LA Nicotina, escala de FAgerström

(EDNF). InStituto Nacional de Enfermedades Respiratorias. MéXICO, 2003

Ítem $R M$

$1 C 95 \%$

\begin{tabular}{|c|c|c|}
\hline \multicolumn{3}{|l|}{ Ansiedad } \\
\hline Edad & 0.97 & $0.93-1.01$ \\
\hline Sexo & 0.67 & $0.31-1.43$ \\
\hline EDNF & 1.21 & $1.03-1.43$ \\
\hline \multicolumn{3}{|l|}{ Depresión } \\
\hline Edad & $0-98$ & $0.95-1.01$ \\
\hline Sexo & 2.03 & $1.10-3.75$ \\
\hline$E D N F>6$ vs $E D N F<4$ & 2.75 & $1.07-7.04$ \\
\hline \multicolumn{3}{|l|}{ Deseos de fumar } \\
\hline Edad & 0.97 & $0.92-1.02$ \\
\hline Sexo & 2.19 & $0.69-6.98$ \\
\hline EDNF & 1.53 & $1.19-1.97$ \\
\hline \multicolumn{3}{|l|}{ Sudoración } \\
\hline Edad & 1.02 & $0.99-1.05$ \\
\hline Sexo & 0.60 & $0.32-1.13$ \\
\hline$E D N F>6$ vs $E D N F<4$ & 2.73 & $1.07-6.96$ \\
\hline \multicolumn{3}{|l|}{ Malestar gastrointestinal } \\
\hline Edad & 0.96 & $0.93-0.99$ \\
\hline Sexo & 0.81 & $0.44-1.50$ \\
\hline EDNF & 1.12 & $0.97-1.28$ \\
\hline \multicolumn{3}{|l|}{ Aumento del apetito } \\
\hline Edad & 0.96 & $0.93-0.99$ \\
\hline Sexo & 1.03 & $0.54-1.94$ \\
\hline EDNF & 1.13 & $0.98-1.30$ \\
\hline
\end{tabular}

Los participantes fueron reclutados entre enero y noviembre de 2003 en la clínica para dejar de fumar del Instituto Nacional de Enfermedades Respiratorias, México.

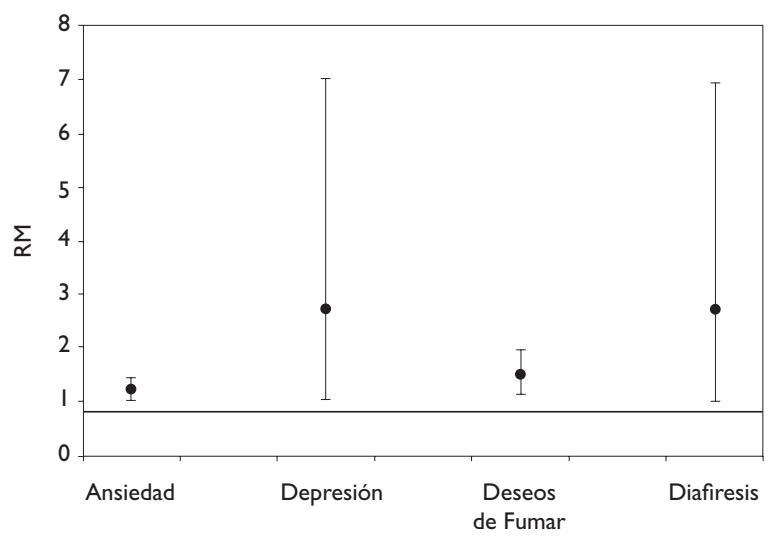

Figura I. RM AJUSTADA POR EDAD Y GÉNERO ENTRE SUJETOS FUMADORES EN FUNCIÓN DEL NIVEL DE DEPENDENCIA A LA Nicotina. Escala de FAgerström. 
concentrarse, irritabilidad, palpitaciones, temblor, mareos, hormigueos y cefalea) no se relacionaron con el nivel de dependencia a la nicotina.

\section{Discusión}

Dejar de fumar parece producir un incremento de s ntomas similares a los reportados por desórdenes de tipo psiquiátricos. Entre los s ntomas más comunes a este tipo de trastornos destacan alteraciones en el estado de ánimo tales como ansiedad y depresión. ${ }^{15}$ Otros s ntomas identificados como indicadores del s ndrome de abstinencia son la dificultad para concentrarse, insomnio e inquietud. ${ }^{12}$

Este es el primer estudio que reporta s ntomas indicadores de abstinencia en un grupo de fumadores mexicanos. De acuerdo con los resultados obtenidos, los s ntomas de abstinencia se presentan en un porcentaje importante de los fumadores en relación con el grupo de no fumadores. En el presente estudio los s ntomas más relacionados con dejar de fumar y dependencia a la nicotina fueron la presencia de deseos de fumar, ansiedad, depresión y diaforesis. A medida que se incrementa la puntuación en la EDNF aumenta el riesgo de desarrollar ansiedad; en las mujeres que dejan de fumar la probabilidad de desarrollar depresión casi se triplica cuando la EDNF es mayor a 6 puntos. El riesgo de presentar deseos de fumar se relaciona con mayores puntajes en la EDNF; finalmente, la sudoración también se asocia positivamente con el nivel de dependencia a la nicotina evaluada mediante la EDNF, especialmente cuando la puntuación es mayor de 6 puntos en comparación con individuos con puntuación menor (cuadro II).

Otros s ntomas son más frecuentes entre los fumadores que dejan de fumar pero no parecen asociarse con la dependencia a la nicotina. Entre éstos destacan el aumento del apetito y malestar gastrointestinal (especialmente entre individuos jóvenes), irritabilidad, tensión, insomnio, mareos, cefalea y dificultad para concentrarse. Otros estudios han informado prevalencias de s ntomas relacionados con s ndrome de abstinencia como tos en $54 \%$, dolor de cabeza en $33 \%$, dificultad para concentrarse en $49 \%$ e inquietud en $64 \%, 11$ los cuales no fueron significativos en nuestro estudio. En un trabajo reciente, Hughes realizó una revisión de la literatura con la finalidad de describir los s ntomas que pueden asumirse como indicadores válidos de la abstinencia a la nicotina, entre los que destacan la ansiedad, depresión, dificultad para concentrarse, insomnio e inquietud. ${ }^{12,16}$ Llamó la atención que entre las mujeres fumadoras el riesgo de desarrollar depresión fue mayor en comparación con los hombres. A este respecto, Leventhal y colaboradores ${ }^{17}$ estudiaron las diferencias en la expresión de s ntomas de abstinencia entre hombres y mujeres; en ese estudio las mujeres mostraron, en general, algunas diferencias cualitativas en relación con s ntomas relacionados con afecto negativo, sin embargo, no se describen patrones espec ficos de s ntoma de abstinencia en relación con el género. ${ }^{17}$

Asimismo, otros s ntomas como el mareo, fatiga y s ntomas $\mathrm{f}$ sicos diversos no parecen estar en relación con la abstinencia. La mayor a de los modelos estudiados, tanto en animales como en humanos, sugieren que el s ndrome de abstinencia se relaciona con la disminución de la actividad dopaminérgica, lo que explicar a por qué unos s ntomas describen mejor el fenómeno de la suspensión abrupta de la nicotina y disminución en los pulsos de dopamina, mientras que otros s ntomas reflejar an más los aspectos psicológicos de la conducta de fumar. ${ }^{9}$ Se ha observado que la aparición de s ntomas de abstinencia es casi inmediata, y se puede percibir desde los primeros minutos post abandono del tabaco. ${ }^{18}$

Aunque la prevalencia de s ntomas en un grupo de fumadores mexicanos es similar a la reportada por otros estudios, ${ }^{19}$ llama la atención que s ntomas como irritabilidad, aumento del apetito y dificultad para concentrarse, tradicionalmente asociados con el s ndrome de abstinencia, no se asociaron significativamente con la EDNF. Por ejemplo, la Escala de Abstinencia al Cigarro (CWS-21) refiere deseos de fumar, depresión - ansiedad, irritabilidad-, impaciencia, dificultad para concentrarse, aumento de apetito y peso e insomnio, como s ntomas consistentes de abstinencia. ${ }^{19}$ Aunque la Escala de Fagerström ha mostrado su utilidad para evaluar el grado de adicción a la nicotina, ${ }^{20}$ existen estudios que señalan que la EDNF no evalúa correctamente los aspectos de dependencia a la nicotina considerados entre los criterios del DSM-IV y la CIE10 para definir al s ndrome de abstinencia, por lo que el planteamiento de explorar el riesgo de distintos s ntomas en función de la EDNF puede ser erróneo. ${ }^{21}$ Existen en la actualidad diversos instrumentos para evaluar el s ndrome de abstinencia, entre los que se encuentran la Escala Minnesota de Abstinencia a la Nicotina (MNWS), la escala de $S$ ntomas $f$ sicos y de Estado de ánimo (MPSS), la Escala Shiffman (SS), la escala Wisconsin de Abstinencia de Tabaquismo (WSWS) y la recientemente introducida escala de Abstinencia del cigarrillo (CWS). La evaluación de s ntomas de abstinencia con cualquiera de las escalas mencionadas resulta recomendable, en general. Algunas escalas son menos sensibles para detectar algún s ntoma en particular como depresión (MNWS, CWS), insomnio (WSWS, MNWS) o ansiedad (MPSS). ${ }^{19,22,23}$ Las escalas más amplias que incorporan un gran número de tópicos o tems no resultan más confiables o precisas en comparación con las escalas más breves. ${ }^{21}$ 
En el presente trabajo se encontró una asociación significativa entre deseos de fumar, ansiedad, depresión y diaforesis con el nivel de adicción a la nicotina. Otros s ntomas fueron más frecuentes entre los fumadores que entre los no fumadores, por lo que se recomienda recabar su expresión entre los pacientes que intentan dejar de fumar.

Este estudio nos confirma que los pacientes con alto grado de adicción deber an recibir apoyo espec fico, ya sea a base de tratamiento farmacológico, tratamiento psicológico enfocado a la modificación de la conducta o la combinación de ambos con la finalidad de reducir los s ntomas de abstinencia. El s ndrome de abstinencia se asocia con un deterioro importante desde el punto de vista cl nico, el cual puede producir algún grado de discapacidad en los fumadores que dejan de fumar. El desarrollo de s ntomas de abstinencia, y especialmente de depresión, se asocia con mayor riesgo de reca da, ${ }^{12}$ lo que provoca una disminución del éxito de los programas para dejar de fumar o en las consultas médicas. El terapeuta, médico y el propio paciente se sienten frustrados cuando éste vuelve a fumar o no puede dejar de fumar. Los pacientes con alta dependencia a la nicotina requieren de múltiples métodos para la cesación del tabaquismo, los cuales deben de ser efectivos y comprobados. A partir de los datos presentados, será necesario adaptar los tratamientos para dejar de fumar a fin de aumentar los éxitos de abstinencia y disminuir las reca das.

\section{Referencias}

I.World Health Organization. The smoking epidemic: a fire in the global village. 25th August, Press Release WHO/ 6I. Geneve:WHO, 1997. 2. Secretaría de Salud. Dirección General de Epidemiología. Dirección General de Estadística e Informática. México: SSA, 2004. 3. Secretaría de Salud. Consejo Nacional Contra las Adicciones (CONADIC). Encuesta Nacional de Adicciones 2002. Tabaco, alcohol y otras drogas. Resumen Ejecutivo. México: SSA, 2002.

4. US Department of Health and Human Services. Reducing the health consequences of smoking. 25 years of progress. A Report of the Surgeon General.Washington, DC: US Government Printing Office, 1989.
5. Kuri PM,Alegre DJ, Mata MA, Hernández AM. Mortalidad atribuible al consumo de tabaco en México. Salud Publica Mex 2002; 44 (Suplemento I):S 29-33.

6. Shoaib M, Schindler CW, Goldberg SR. Nicotine self-administration in rats: strain and nicotine pre-exposure effects on acquisition. Psychopharmacology 1997; 129:35-43.

7. Henningfield D, Goldberg SR. Nicotine as reinforcement in human subjects and laboratory animals. Pharmacol Biochem Behav 1983;19:989992.

8. American Psychiatric Association. Diagnostic and Statistical Manual of Mental Disorders. 4th edition,(DSM-IV). Arlington, Va:APA, 1994.

9. Hughes JR. Effects of abstinence from tobacco: etiology, animal models, epidemiology, and significance: a subjective review. Nicotine Tob Res 2007;9(3):329-39.

I0. Hughes JR, Gust SW, Skoog K, Keenan RM, Fenwick JW. Symptoms of tobacco withdrawal: a replication and extension. Arch Gen Psychiatry |99|;48:52-59.

II.Ward MM, Swan GE, Jack LM. Self- reported abstinence effects in the first month after smoking cessation. Addictive Behaviors 200 I;26:31 I-327. 12. Hughes JR. Effects of abstinence from tobacco: valid symptoms and time course. Nicotine Tob Res 2007;9(3):315-327.

13. Organización Mundial de la Salud. Décima Revisión de la Clasificación Internacional de las Enfermedades (CIEIO). Trastornos Mentales y del Comportamiento. Madrid: Forma, 1993.

14. Goldberg DP. Standardized Psychiatric Interview (SPI) Institute of Psychiatry, 1969. Cuadernos Científicos CEMESAM II 1979:49-50.

15. Hughes JR. Clinical significance of tobacco withdrawal. Nicotine and Tobacco Research 2006;8(2):153-156.

16. Camí J, Farré M. Drug addiction. N Engl J Med 2003; 349:975-986. 17. Leventhal AM, Waters AJ, Boyd S, Moolchan ET, Lerman C,

Pickworth WB. Gender differences in acute tobacco withdrawal: effects on subjective, cognitive, and physiological measures. Exp Clin Psychopharmacol 2007; I5(I):21-36.

18. Hendricks PS, Ditre JW, Drobes DJ, Brandon TH. The early time course of smoking withdrawal effects. Psychopharmacology (Berl) 2006; 187(3):385-396.

19. Etter JF.A self- administered questionnaire to measure cigarette withdrawal symptoms:The Cigarette Withdrawal Scale. Nicotine Tob Res 2005; 7(I):47-57.

20. Fagerström KO, Schneider NG. Measuring nicotine dependence:A review of the Fagerström Tolerance Questionnaire. Journal of Behavioral Medicine 1989;12: 159-181.

2I. Etter JF.A comparison of the content-, construct- and predictive validity of the cigarette dependence scale and the Fagerstrom test for nicotine dependence. Drug Alcohol Depend 2005; 7;77(3):259-268.

22. West R, Ussher M, Evans M, Rashid M. Assessing DSM-IV nicotine withdrawal symptoms: a comparison and evaluation of five different scales. Psychopharmacology (Berl) 2006;184(3-4):619-627.

23. Cappelleri JC, Bushmakin AG, Baker CL, Merikle E, Olufade AO, Gilbert DG. Revealing the multidimensional framework of the Minnesota nicotine withdrawal scale. Curr Med Res Opin 2005;2I(5):749-760. 